\title{
Magnetic flows and Gaussian thermostats on manifolds of negative curvature
}

\author{
by
}

\author{
Maciej P. Wojtkowski (Tucson, AZ)
}

\begin{abstract}
We consider a class of flows which includes both magnetic flows and Gaussian thermostats of external fields. We give sufficient conditions for such flows on manifolds of negative sectional curvature to be Anosov.
\end{abstract}

0. Introduction. The geodesic flow on a Riemannian manifold describes inertial motion of a point particle confined to the manifold. If the manifold has negative sectional curvature we obtain an Anosov flow, a prime example of a dynamical system with good statistical properties. In the present paper we study flows generated by special forces, magnetic flows and Gaussian thermostats.

Magnetic flows in this context were discussed already 30 years ago by Anosov and Sinai [A-S]. They were studied recently by Gouda [Go], Grognet $[\mathrm{Gr}]$, and M. and P. Paternain [P-P]. Just like the geodesic flow the magnetic flow lives naturally on the unit tangent bundle.

Another class of flows on the unit tangent bundle was introduced recently in physics literature, the Gaussian thermostat of an external field $[\mathrm{H}]$.

We show (Section 1) that both classes of flows can be represented as special cases of a general construction. We define a generalized magnetic flow on the tangent bundle $T M$ (or the cotangent bundle $T^{*} M$ ) by requiring that its velocity vector field $F$ satisfies

$$
i_{F}(\omega-\gamma)=-d H,
$$

where $\omega$ is the standard symplectic form, $H$ is a hamiltonian and the 2-form $\gamma$ represents the generalized magnetic field.

2000 Mathematics Subject Classification: 37D20, 37D40.

I thank François Ledrappier, Feliks Przytycki, Marek Rychlik and Lai-Sang Young who generously shared with me their knowledge of SRB measures. 
This relation is classical for magnetic flows, where $\gamma$ is a closed 2-form on $M$. In general, although it resembles Hamiltonian formalism, it does not have the usual consequences. However, we show that for any 2-form $\gamma$ on $M$ the resulting flow is volume preserving. It seems that this fact was overlooked in the papers on magnetic flows.

For the Gaussian thermostats the relation (0.1) appeared in [W-L] and it was the basis for representing the flow as a conformally symplectic system. In this paper we are making the point that (0.1) holds because the Gaussian thermostat can be viewed as a velocity dependent magnetic field. We discuss these results in Section 2 and compare them with the work of Dettmann and Morriss [D-M].

In Section 3 we consider general flows with potential fields, magnetic fields and Gaussian thermostats. We show how such systems look like in Darboux coordinates and we calculate the divergence of the resulting vector field. This generalizes the coordinate free calculations of Sections 1 and 2 .

We discuss the average rate of volume contraction which was shown by Ruelle $[R]$ to represent the average rate of entropy production in the system. Its positivity (= dissipativity of the system) is usually hard to establish in concrete examples. In the case of surfaces of constant negative curvature Gaussian thermostats were studied by Bonetto, Gentile and Mastropietro [B-G-M], and they addressed the problem of dissipativity. We show (Proposition 3.1) that if the flow is Anosov and the external field has a local potential but no global potential then the system is dissipative. We can allow magnetic fields but no "internal" potential interactions. It would be interesting to understand also this "isoenergetic" case. The proof of Proposition 3.1 is deceptively simple because it relies heavily on the deep results of Ledrappier and Young $[\mathrm{L}-\mathrm{Y}]$.

In the rest of the paper we address the question of when the general flow on a manifold with negative sectional curvature is Anosov. Gouda [Go] and Grognet $[\mathrm{Gr}]$ obtained sufficient conditions for magnetic flows. We obtain similar conditions for the general flows (Theorem 4.1 and its corollaries).

The proof of Theorem 4.1 (Section 6) is based on a criterion for Anosov property, formulated and proven in Section 5, involving indefinite quadratic forms. This criterion generalizes to Anosov flows a result of Lewowicz [L] on Anosov diffeomorphisms (see also [K-B] and [W]). Important technical advantage of this method is that we can do calculations in the ambient space, although they relate to a level set of the hamiltonian.

1. Potential flows, magnetic flows and Gaussian thermostats. Let us consider a compact $n$-dimensional Riemannian manifold $M$ and its tangent bundle $\pi: T M \rightarrow M$. For a smooth function $W: M \rightarrow \mathbb{R}$ we 
introduce the potential flow on $T M$ defined by the equations

$$
\frac{d q}{d t}=v, \quad \frac{D v}{d t}=-\nabla W,
$$

where $q \in M$ and $D / d t$ denotes the covariant derivative. Let $\omega$ be the canonical symplectic form transported from $T^{*} M$ to $T M$ by the natural isomorphism. The vector field $F$ on $T M$ defined by (1.1) is a Hamiltonian vector field with hamiltonian $H=\frac{1}{2} v^{2}+W$ and we have $i_{F} \omega=-d H$.

We are going to modify (1.1) by adding "nonpotential forces". A 2-form $\gamma$ on $T M$ will be called a generalized magnetic field (gmf) if there is an antisymmetric operator $Y$ on the tangent spaces on $M$ (depending in general on the point in $T M$ ) such that

$$
\langle Y d \pi \cdot, d \pi \cdot\rangle=\gamma(\cdot, \cdot) .
$$

We consider the generalized magnetic flow on $T M$ defined by

$$
\frac{d q}{d t}=v, \quad \frac{D v}{d t}=-\nabla W+Y v .
$$

Now the vector field $F$ on $T M$ defined by (1.3) satisfies

$$
i_{F} \Omega=-d H, \quad \text { where } \quad \Omega=\omega-\gamma .
$$

To prove this let us introduce the identification of the tangent space of $T M$ at $(q, v)$ with $T_{q} M \oplus T_{q} M$. For a tangent vector defined by a parametrized curve $(q(u), v(u)),|u|<\varepsilon$, we use $(\xi, \eta) \in T_{q(0)} M \oplus T_{q(0)} M$ with

$$
\xi=\frac{d q}{d u}, \quad \eta=\frac{D v}{d u}
$$

as the linear coordinates. In these coordinates $F=(v,-\nabla W+Y v)$ and the symplectic form is $\omega\left(\left(\xi_{1}, \eta_{1}\right),\left(\xi_{2}, \eta_{2}\right)\right)=\left\langle\xi_{2}, \eta_{1}\right\rangle-\left\langle\xi_{1}, \eta_{2}\right\rangle$. We have further

$$
\begin{aligned}
\omega(F,(\xi, \eta)) & =-\langle v, \eta\rangle+\langle-\nabla W+Y v, \xi\rangle, \\
\gamma(F,(\xi, \eta)) & =\langle Y v, \xi\rangle, \\
d H((\xi, \eta)) & =\langle v, \eta\rangle+\langle\nabla W, \xi\rangle,
\end{aligned}
$$

which proves (1.4).

$\Omega$ is always nondegenerate because, roughly speaking, $\gamma$ depends on $d q$ but not on $d v$, so it cannot destroy the nondegeneracy of $\omega$. However $\Omega$ is closed only if $\gamma$ is closed. When $\Omega$ is not closed (1.4) does not amount to much because it does not force the preservation of $\Omega$ by the flow, which is the cornerstone of Hamiltonian formalism.

Special cases of this construction include the magnetic flow studied by Gouda [Go], Grognet [Gr], and M. and G. Paternain [P-P]. In this case the form $\gamma$ is the pullback under the projection $\pi$ of a 2 -form $\widehat{\gamma}$ on $M$. In view of (1.4), if $\widehat{\gamma}$ is closed (which is a natural assumption for a magnetic field), the magnetic flow is a Hamiltonian flow with hamiltonian $H$ with respect to 
a modified symplectic structure. If $\widehat{\gamma}$ is not closed the resulting flow is not, in general, symplectic with respect to any symplectic structure. It turns out that without any assumptions on $\widehat{\gamma}$ the magnetic flow preserves the Lebesgue measure on $T M$ and hence for such flows we get smooth invariant measures on the level sets of $H$ (energy shells).

Proposition 1.5. If $\gamma$ is a pullback to TM of a 2-form $\widehat{\gamma}$ on $M$ and a vector field $F$ on $T M$ satisfies

$$
i_{F} \omega-i_{F} \gamma=-d H
$$

for a smooth function $H$ on $T M$ then the flow defined by the vector field $F$ preserves the standard Lebesgue measure on TM (the symplectic volume element).

In the proof we will use the following observation:

Lemma 1.6. If a $k$-form $\zeta$ on $T^{*} M$ is the pullback of a $k$-form $\widehat{\zeta}$ defined on $M$ then

$$
\zeta \wedge \omega^{\wedge l}=0 \quad \text { for } l \geq n-k+1 .
$$

As a consequence we obtain $(\omega-\gamma)^{\wedge n}=\omega^{\wedge n}$. We proceed with the calculation of the Lie derivative:

$$
\begin{aligned}
\mathcal{L}_{F} \omega^{\wedge n} & =\mathcal{L}_{F}(\omega-\gamma)^{\wedge n}=n d\left(i_{F}(\omega-\gamma) \wedge(\omega-\gamma)^{\wedge(n-1)}\right) \\
& =-n d\left(d H \wedge(\omega-\gamma)^{\wedge(n-1)}\right) \\
& =-n(n-1) d H \wedge d \gamma \wedge(\omega-\gamma)^{\wedge(n-2)} .
\end{aligned}
$$

But $d \gamma \wedge(\omega-\gamma)^{\wedge(n-2)}=0$ by Lemma 1.6. Proposition 1.5 is proven. We will obtain a different, coordinate proof in Section 3.

Another interesting example of a generalized magnetic field is an external field with the Gaussian thermostat which we are going to describe in detail. Let $E$ be a vector field on $M$, for example the gradient vector field $E=-\nabla U$ for some potential function $U$. We consider the flow in $T M$ given by

$$
\frac{d q}{d t}=v, \quad \frac{D v}{d t}=-\nabla W+E .
$$

The resulting flow does not in general preserve the energy function $H=$ $\frac{1}{2} v^{2}+W$. We impose the preservation of $H$ via the Gauss least constraint principle $[\mathrm{H}]$. The resulting equations are

$$
\frac{d q}{d t}=v, \quad \frac{D v}{d t}=-\nabla W+E-\frac{\langle E, v\rangle}{v^{2}} v .
$$

These systems appear in physics literature under the name of "isoenergetic dynamics". They fit our formalism of generalized magnetic fields. 
We introduce the 2 -form $\gamma$ by

$$
\alpha((\xi, \eta))=\langle v, \xi\rangle, \quad \Upsilon((\xi, \eta))=\langle E, \xi\rangle, \quad \gamma=\frac{1}{v^{2}} \alpha \wedge \Upsilon .
$$

Note that $d \alpha=\omega$ and in the special case of the potential vector field $E=$ $-\nabla U$, we have $\Upsilon=-d U$ (strictly speaking $\Upsilon=-\pi^{*} d U$, but we will allow this kind of identification). The antisymmetric operator

$$
Y(\cdot)=\frac{1}{v^{2}}(\langle v, \cdot\rangle E-\langle E, \cdot\rangle v)
$$

satisfies (1.2) and the corresponding generalized magnetic flow on $T M$ coincides with (1.7).

2. Isokinetic dynamics and conformally symplectic structures. Let us look more carefully at the Gaussian thermostat of an external field $E$ when $W \equiv 0$, i.e., $H=\frac{1}{2} v^{2}$. It is called the isokinetic dynamics. The derivative $d \gamma$ does not vanish (except in trivial cases). If the form $\Upsilon$ is closed (locally $\Upsilon=-d U$ ), then for a fixed level set $v^{2}=c$ we can modify the form $\gamma$ to $\gamma_{c}=\frac{1}{c} \alpha \wedge \Upsilon$ without changing the gmf on the level set. Now we have

$$
d \Omega_{c}=d\left(\omega-\gamma_{c}\right)=\frac{1}{c} d U \wedge \omega=\frac{1}{c} d U \wedge \Omega_{c} .
$$

The significance of $(2.1)$ is revealed when we calculate $d\left(e^{-U / c} \Omega_{c}\right)=0$, which shows that $e^{-U / c} \Omega_{c}$ defines a genuine symplectic structure. In such a situation we say that the form $\Omega_{c}$ defines a conformally symplectic structure. It follows that when the field $E$ has a global potential $U$ the resulting flow on the level set $v^{2}=c$ is a Hamiltonian flow with respect to the symplectic structure $e^{-U / c} \Omega_{c}$ and the hamiltonian $e^{-U / c}\left(v^{2} / 2-c / 2\right)$. Indeed on the level set $v^{2}=c$ we have

$$
i_{F}\left(e^{-U / c} \Omega_{c}\right)=-d\left(e^{-U / c}\left(v^{2} / 2-c / 2\right)\right) .
$$

In particular when the field $E$ has a global potential $U$ the flow has a smooth invariant measure. We will obtain the density of this invariant measure at the end of this section and then again, by coordinate calculations, in Section 3.

This geometric setup for the Gaussian thermostat of a locally potential field $E$ was discovered in [W-L]. It explains the symmetry of Lyapunov spectrum which was first observed numerically by Evans, Cohen and Morriss $[\mathrm{E}-\mathrm{C}-\mathrm{M}]$, and then proved by Dettmann and Morriss [D-M]. In their proof they consider tangent subspaces transversal to the flow, defined by the condition that $\xi$ be orthogonal to $v$. This tangent subbundle is not invariant under the flow. (We should not expect in general any invariant geometrically defined transversal subbundle, the case of a geodesic flow, a contact flow, is in this respect rather special.) They consider the projection of the linearized flow onto this subbundle and observe that the linear equations 
are infinitesimally Hamiltonian up to addition of a multiple of identity. This situation can be understood in our language in the following way.

Let us calculate the Lie derivative of $\Omega_{c}$ in the direction of the vector field $F$ of our flow. Using the Cartan formula we obtain

$$
\mathcal{L}_{F} \Omega_{c}=\frac{1}{c}\left(d U(F) \Omega_{c}+d U \wedge d H\right)
$$

This is not a nice formula in general because it shows that such flows do not preserve the conformally symplectic structure. That is probably why in his geometric study of conformally symplectic dynamics Vaisman [V] defined Hamiltonian flows differently, and his Hamiltonian flows do preserve the structure. However, if we restrict the flow to the level set of the hamiltonian, $v^{2}=c$ (which we have already done anyway), we obtain

$$
\mathcal{L}_{F} \Omega_{c}=\frac{1}{c} d U(F) \Omega_{c} .
$$

Hence under the action of the flow the restriction of the form $\Omega_{c}$ to the level set will be multiplied by $e^{\left(U_{1}-U_{0}\right) / c}$ when we move from the value $U_{0}$ of the potential to the value $U_{1}$. The restriction of the form $\Omega_{c}$ projects onto the quotient tangent space (quotient by the span of the velocity vector field). When we restrict $\Omega_{c}$ further to the Dettmann-Morriss transversal subspace ( $\xi$ orthogonal to $v$ ) we see that the form $\alpha$ vanishes and we obtain the restriction of the canonical symplectic form $\omega$. Now (2.2) captures the essence of the Dettmann-Morriss proof.

Similarly to the symplectic case we can consider the $k$ th exterior power of the form $\Omega_{c}$ and again its restriction to the level set is preserved up to a scalar factor $e^{k\left(U_{1}-U_{0}\right) / c}$. The $(n-1)$ th exterior power defines a volume element on the quotient transversal tangent subspace. This transversal volume element can be further uniquely extended to the full volume element $\Xi$ on the level set by the condition

$$
i_{F} \Xi=\Omega_{c}^{\wedge(n-1)} .
$$

A moment of reflection convinces us that because on the orthogonal transversal subspace the form $\Omega_{c}$ coincides with the standard symplectic form, $\Xi$ is (up to a constant factor) the standard Lebesgue volume element on the sphere bundle (the level set of $H$ ). Moreover under the action of the flow this volume element will be multiplied by the same coefficient as the form $\Omega_{c}^{\wedge(n-1)}$, i.e., $e^{(n-1)\left(U_{1}-U_{0}\right) / c}$. This follows from a simple calculation using the Cartan formula and (2.2). We will also obtain a simple coordinate proof in the next section.

We conclude that if the field $E$ has a global potential $U$ then the resulting flow preserves the smooth invariant measure which has the density $e^{-(n-1) U / c}$ with respect to the standard volume element on the sphere bundle $v^{2}=c$. 
It was recently shown by Bonetto, Cohen and Pugh [B-C-P] with a careful numerical study that in general if $W \neq 0$, i.e., in the case of isoenergetic dynamics, the Floquet exponents do not obey the shifted symplectic symmetry. Thus there is no conformally symplectic structure preserved by the flow.

3. The general flow and entropy production. Let us now combine a potential field, a magnetic field and an external field with the Gaussian thermostat, i.e., we consider equations of the form

$$
\frac{d q}{d t}=v, \quad \frac{D v}{d t}=-\nabla W+B v+E-\frac{\langle E, v\rangle}{v^{2}} v .
$$

Now the vector field $F$ defined by (3.1) is the gmf with respect to $\gamma=$ $\beta+\frac{1}{v^{2}} \alpha \wedge \Upsilon$, where $B$ and $\beta$ describe the magnetic field. In this case, even if $W \equiv 0$ and $\beta$ is closed, we do not in general have a conformally symplectic structure. We will calculate the divergence of the vector field to be $-(n-1) v^{-2}\langle E, v\rangle$, equal to $(n-1) v^{-2} d U / d t$ in the potential case. It follows that in the isokinetic case $W \equiv 0$, if the external field has a global potential $U$ the flow preserves the smooth invariant measure with density $e^{-(n-1) U / v^{2}}$ with respect to the standard volume element. It seems that in the isoenergetic case $W \neq 0$, even if the external field has a global potential the flow may have no absolutely continuous invariant measure, because now $v^{2}$ is not constant. It would be interesting to have an explicit example.

We will do the calculations in standard symplectic coordinates in $T^{*} M$. Now $\omega=\sum d p \wedge d q$ denotes the standard symplectic form in $T^{*} M$ and the gmf is defined by the form $\gamma=\sum_{k, l} c_{k l} d q_{k} \otimes d q_{l}$ where the matrix of coefficients $C=\left\{c_{k l}\right\}$ is antisymmetric. A vector field $F$ on $T^{*} M$ which satisfies $i_{F} \omega-i_{F} \gamma=-d H$ for a smooth function $H$ on $T^{*} M$ generates the following differential equations:

$$
\dot{q}=\frac{\partial H}{\partial p}, \quad \dot{p}=-\frac{\partial H}{\partial q}-C \frac{\partial H}{\partial p} .
$$

We can immediately calculate the divergence of the vector field (3.2). We obtain

$$
\operatorname{div} F=-\sum_{k, l} \frac{\partial c_{k l}}{\partial p_{k}} \frac{\partial H}{\partial p_{l}} .
$$

It follows that for magnetic flows the divergence is zero because the entries of $C$ are functions on $M$. This provides another proof of Proposition 1.5.

In the case of the flow (3.1) we obtain

$$
c_{k l}=\frac{1}{p^{2}}\left(p_{k} E_{l}-p_{l} E_{k}\right)
$$


where $\Upsilon=\sum E_{k} d q_{k}$. Hence

$$
\operatorname{div} F=\frac{1}{p^{2}} \sum_{k, l} c_{k l} \frac{\partial p^{2}}{\partial p_{k}} \frac{\partial H}{\partial p_{l}}-\frac{1}{p^{2}} \sum_{k} \sum_{l \neq k} E_{l} \frac{\partial H}{\partial p_{l}} .
$$

The first sum vanishes if $H=\frac{1}{2} p^{2}+W(q)$ for some function $W$ (the case of isoenergetic dynamics), and the second term yields

$$
\operatorname{div} F=-\frac{1}{p^{2}} \sum_{k}\left(\langle E, \dot{q}\rangle-E_{k} \dot{q}_{k}\right)=\frac{-(n-1)}{p^{2}}\langle E, \dot{q}\rangle .
$$

In the potential case $\Upsilon=-d U$ we get $(n-1) p^{-2} d U / d t$.

If the external field $E$ is nonvanishing then in the flow (3.1) we can expect that asymptotically the velocity $v$ is aligned with the field $E$ and hence that asymptotically the phase volume is contracted. This intuition is supported by the following considerations. Let us denote by $\phi^{t}=\phi_{h}^{t}$ our flow on the energy shell $H=h$ (we assume that $h$ is large enough so that $v^{2}$ does not vanish) and by $\lambda$ the normalized volume element on this energy shell. Suppose that the flow has an asymptotic invariant measure $\mu$ equal to the weak limit of the time averages $T^{-1} \int_{0}^{T} \phi_{*}^{t} \lambda d t$ as $T \rightarrow+\infty$ (possibly on a subsequence). Now $e=-\int \operatorname{div} F d \mu$ represents the average logarithmic rate of volume contraction in this asymptotic state. As shown by Ruelle $[R]$, this quantity can be interpreted as the average rate of entropy production in the system and it is always nonnegative. It is interesting to investigate conditions under which $e$ is positive. One such case is a nonvanishing strong field $E$. Indeed we can calculate

$$
\frac{d}{d t}\langle E, v\rangle=\left\langle\nabla_{v} E, v\right\rangle-\langle E, \nabla W\rangle+\langle E, B v\rangle+E^{2}-\frac{1}{c}\langle E, v\rangle^{2},
$$

and we see that where $\langle E, v\rangle=0$ we have $\frac{d}{d t}\langle E, v\rangle>0$ if only $E \neq 0$ and we multiply it by a sufficiently large scalar. It is not hard to prove that in such a case the support of $\mu$ is contained in the domain $\langle E, v\rangle>0$. This follows from the fact that in the domain $\langle E, v\rangle<0$ the volume is expanded under the flow.

In the rest of this section we consider the isokinetic case $W \equiv 0$. If the external field $E$ has a global potential then the system has a smooth invariant measure. A more interesting situation arises when the external field has a local potential but no global potential ( $\Upsilon$ is closed but not exact). Let us again consider the asymptotic measure $\mu$ as introduced above. Now the positivity of $e$ can also be interpreted as the establishment of current in the system since by $(3.3), e=(n-1) v^{-2} \int\langle E, v\rangle d \mu$.

In the case of surfaces of constant negative curvature Gaussian thermostats were studied by Bonetto, Gentile and Mastropietro [B-G-M] and they addressed the question of positivity of $e$. Let us consider more gener- 
ally a Riemannian manifold with negative sectional curvature. By structural stability if the magnetic and external fields are sufficiently small we obtain an Anosov flow topologically conjugate to the geodesic flow $[\mathrm{K}-\mathrm{H}]$. Moreover the topological conjugacy is homotopic to identity. Such flows have unique asymptotic measures, called SRB measures [R]. We have

Proposition 3.1. If the flow $\phi$ is an Anosov flow topologically conjugate to the geodesic flow, the conjugacy is homotopic to identity and the external field has a local but no global potential then the entropy production e with respect to the $S R B$ measure $\mu$ is positive.

Pr o of. We begin by repeating the argument given by Ruelle in the case of discrete time $[\mathrm{R}]$. Let us assume to the contrary that $e=-\int \operatorname{div} F d \mu=0$. Then the sum of positive Lyapunov exponents (with respect to $\mu$ ) is equal to the sum of negative Lyapunov exponents and hence the metric entropy of $\phi^{-1}$ satisfies the Pesin formula. It follows from the work of Ledrappier and Young [L-Y] that the measure $\mu$ is absolutely continuous on both stable and unstable foliations and hence it is absolutely continuous. Moreover the density with respect to the volume element $\lambda$ must be positive and continuous.

Such an invariant measure contradicts the multivaluedness of the potential $U$. Indeed let us take a closed loop $l$ on $M$ such that $\oint_{l} d U>0$. There is a periodic geodesic $l^{\prime}$ homotopic to $l$. Further by topological conjugacy our flow has a periodic orbit $\zeta$ with projection on $M$ homotopic to $l^{\prime}$ and $l$. The time integral $\oint_{\zeta} \operatorname{div} F d t$ of the divergence along the periodic orbit is equal to $-(n-1)(2 h)^{-1} \oint_{l} d U<0$. On the other hand it is equal to the logarithm of the Jacobian of $\phi^{T}$ on our T-periodic orbit. Since the flow has an absolutely invariant measure with continuous positive density the Jacobian must be equal to 1.

This proposition raises the question of explicit conditions on the magnetic and external fields that imply the Anosov property. The rest of the paper is devoted to this problem.

4. When is the general flow Anosov? Gouda [G] and Grognet [Gr] obtained conditions on the magnetic field on a manifold of negative sectional curvature which imply that the flow is Anosov. We will extend their results by including potential fields, external fields and Gaussian thermostats. Hence we consider the general flow defined by the equations

$$
\frac{d q}{d t}=v, \quad \frac{D v}{d t}=-\nabla W+B v+E-\frac{\langle E, v\rangle}{v^{2}} v,
$$

where $W$ is a smooth function on $M$ (the potential energy of internal interactions), $B$ is a magnetic field, and $E$ is the external field. We restrict the 
system to the level set $h=\frac{1}{2} v^{2}+W$ and we assume that $h$ is large enough so that $v$ does not vanish. We denote by $R(\cdot, \cdot)$ the curvature tensor.

THEOREM 4.1. If for every point $q \in M$ and $v \in T_{q} M$ with $v^{2}=$ $2(h-W)$, the quadratic form

$-\langle R(\xi, v) v, \xi\rangle-\left\langle\nabla_{\xi} \nabla W, \xi\right\rangle+\left\langle\nabla_{\xi} B v, \xi\right\rangle+\left\langle\nabla_{\xi} E, \xi\right\rangle$

$-\frac{\langle E, v\rangle^{2}}{4 v^{4}} \xi^{2}-\frac{1}{4}(B \xi)^{2}+\frac{1}{4 v^{2}}\langle\xi,-2 \nabla W+B v\rangle^{2}-\frac{1}{v^{2}}\langle\xi,-2 \nabla W+B v+E\rangle^{2}$ in $\xi \in T_{q} M$ is positive definite on the subspace of $T_{q} M$ orthogonal to $v$, then the system is Anosov.

The condition in the theorem is clearly satisfied if the sectional curvatures are negative and $h$ is sufficiently large. It can be made less cumbersome by considering special cases. Let the sectional curvatures of $M$ be bounded above by $-k^{2}<0$.

1. Pure potential flow $(B=0, E=0)$. If

$$
\sup _{q \in M}\left(\frac{\left\|\nabla^{2} W\right\|}{2(h-W)}+3\left(\frac{\|\nabla W\|}{2(h-W)}\right)^{2}\right)<k^{2}
$$

then the system is Anosov.

This criterion could be strengthened by the observation that where the potential function is concave $\left(\left\langle\nabla_{\xi} \nabla W, \xi\right\rangle\right.$ negative definite), it adds to the dispersing effect of the negative curvature.

2. Pure magnetic flow $\left(W=0, E=0, v^{2}=c\right)$. If

$$
\frac{\|\nabla B\|}{\sqrt{c}}+\left(\frac{\|B\|}{\sqrt{c}}\right)^{2}<k^{2}
$$

then the system is Anosov.

We have thus obtained the condition of Gouda [Go] and Grognet [Gr] (Grognet does not get optimal coefficients).

3. Pure Gaussian thermostat $\left(W=0, B=0, v^{2}=c\right)$. If

$$
\frac{\|\nabla E\|}{c}+\left(\frac{\|E\|}{c}\right)^{2}<k^{2}
$$

then the system is Anosov.

4. Gaussian thermostat with magnetic field $\left(W=0, v^{2}=c\right)$. If

$$
\frac{\|\nabla B\|}{\sqrt{c}}+\frac{\|\nabla E\|}{c}+\left(\frac{\|B\|}{\sqrt{c}}+\frac{\|E\|}{c}\right)^{2}<k^{2}
$$

then the system is Anosov. 
5. A criterion for a flow to be Anosov. Let $N$ be a smooth compact $k$-dimensional manifold with an auxiliary Riemannian metric. Let further $F$ be a nonvanishing smooth vector field on $N$. We consider the flow $\phi^{t}, t \in \mathbb{R}$, defined by the vector field $F$.

The flow $\phi^{t}, t \in \mathbb{R}$, is Anosov (cf. [K-H]) if there is a $\phi^{t}$-invariant splitting of the tangent bundle $T_{x} M=E_{x}^{0} \oplus E_{x}^{+} \oplus E_{x}^{-}, x \in M$, and two positive constants $a, b$ such that

(1) $E_{x}^{0}=\operatorname{span}\{F(x)\}, x \in M$,

(2) $\left\|D \phi^{\mp t} v\right\| \leq b e^{-t a}\|v\|$ for $v \in E_{x}^{ \pm}$and $t \geq 0$.

The linear subspaces of the splitting are called neutral, unstable and stable, respectively.

In addition to the tangent bundle $T M$ we consider the quotient bundle $\widehat{T} M$ defined by $\widehat{T}_{x} M=T_{x} M / \operatorname{span}\{F(x)\}$, i.e., the linear space $\widehat{T}_{x} M$ is the linear quotient of $T_{x} M$ by the one-dimensional subspace spanned by the vector $F(x)$. The quotient bundle inherits the scalar product and the norm. Since $D_{x} \phi^{t} F(x)=F\left(\phi^{t} x\right)$ we can project the linear map

$$
D_{x} \phi^{t}: T_{x} M \rightarrow T_{\phi^{t} x} M
$$

to the quotient linear spaces $\widehat{T}_{x} M$ and $\widehat{T}_{\phi^{t} x} M$. We denote the projected map by

$$
A_{x}^{t}: \widehat{T}_{x} M \rightarrow \widehat{T}_{\phi^{t} x} M .
$$

The Anosov property can be reformulated in the language of the quotient bundle.

Proposition 5.1. The flow $\phi^{t}, t \in \mathbb{R}$, is Anosov if and only if there is an $A^{t}$-invariant splitting of the quotient bundle $\widehat{T}_{x} M=\widehat{E}_{x}^{+} \oplus \widehat{E}_{x}^{-}, x \in M$, and two positive constants $a, b$ such that

$$
\left\|A_{x}^{\mp t} v\right\| \leq b e^{-t a}\|v\| \quad \text { for } v \in \widehat{E}_{x}^{ \pm} \text {and } t \geq 0 .
$$

Proof. The only thing that needs to be done is the reconstruction of the (un)stable subspace from its projection on the quotient space. We will do it for the stable subspace $\widehat{E}_{x}^{-}$.

We identify the quotient tangent space $\widehat{T}_{x} M$ with the orthogonal complement of $\operatorname{span}\{F(x)\}$. Let $\Pi: T M \rightarrow \widehat{T} M$ be the orthogonal projection and let $\mathcal{L}_{F} \Pi=\left.\frac{d}{d s} D \phi^{-s} \Pi D \phi^{s}\right|_{s=0}$ be its Lie derivative. Consider the linear functional $\lambda(x)$ defined on $\widehat{E}_{x}^{-}$by

$$
\lambda(x ; v)=\int_{0}^{\infty} \frac{\left\langle F,\left(\mathcal{L}_{F} \Pi\right) A_{x}^{s} v\right\rangle}{\langle F, F\rangle} d s .
$$

The integral converges thanks to the decay of $A_{x}^{t} v$, and for any vector $v \in \widehat{E}_{x}^{-}$ 
we have

$$
D \phi^{t}(v+\lambda(x ; v) F(x))=A^{t} v+\lambda\left(\phi^{t} x ; A^{t} v\right) F\left(\phi^{t} x\right) .
$$

It follows that the stable subspace is given by

$$
E_{x}^{-}=\left\{w=v+\lambda(x ; v) F(x) \mid v \in \widehat{E}_{x}^{-}\right\} .
$$

Let $\mathcal{Q}: T M \rightarrow \mathbb{R}$ be a continuous quadratic form on the tangent bundle, i.e., each $\mathcal{Q}_{x}=\mathcal{Q}_{\mid T_{x} M}$ is a quadratic form on the tangent space $T_{x} M$ and it depends continuously on $x$. We assume further that the form can be projected onto the quotient bundle, i.e., $\mathcal{Q}_{x}(w+a F(x))=\mathcal{Q}_{x}(w)$ for any $w \in T_{x} M$ and any real $a$. We will use the same notation for the projected form. We assume that the projected form is nondegenerate, and hence being continuous it has constant signature $(l, m)(l+m=k-1)$.

We assume that the Lie derivative $\mathcal{L}_{F} \mathcal{Q}(w)=\left.\frac{d}{d t} \mathcal{Q}\left(D \phi^{t} w\right)\right|_{t=0}$ of $\mathcal{Q}$ in the direction of the vector field $F$ is continuous. Clearly it can also be projected onto the quotient space. We say that the flow $\phi^{t}$ is strictly monotone (with respect to a quadratic form $\mathcal{Q}$ ) if the projection of the Lie derivative $\mathcal{L}_{F} \mathcal{Q}$ onto the quotient bundle is positive definite.

The following theorem is a generalization of a result of Lewowicz $[\mathrm{L}]$ to flows.

Theorem 5.2. If a flow is strictly monotone then it is Anosov.

Proof. We will work in the quotient bundle $\widehat{T} M$. We define the bundle of positive cones $C=\{v \in \widehat{T} M \mid \mathcal{Q}(v) \geq 0\}$ associated with the form $\mathcal{Q}$. By $C(x)$ we denote the positive cone at $x \in M$. By $C^{\prime}=\{v \in \widehat{T} M \mid \mathcal{Q}(v) \leq 0\}$ we denote the bundle of negative cones.

By strict monotonicity we have $\mathcal{L}_{F} \mathcal{Q}(v) \geq c_{1}\|v\|^{2}$ and also (by compactness) $|\mathcal{Q}(v)| \leq c_{2}\|v\|^{2}$ for all $v \in \widehat{T} M$ and some positive constants $c_{1}, c_{2}$. It follows that

$$
\frac{d}{d t} \mathcal{Q}\left(A^{t} v\right) \geq c_{1}\left\|A^{t} v\right\|^{2} \geq 2 a\left|\mathcal{Q}\left(A^{t} v\right)\right| \quad \text { where } \quad 2 a=\frac{c_{1}}{c_{2}} .
$$

Hence $A^{t} C \subset \operatorname{int} C \cup\{0\}$ and $A^{-t} C^{\prime} \subset \operatorname{int} C^{\prime} \cup\{0\}$ for any $t>0$. Moreover by integrating the last inequality we obtain

$$
\frac{\mathcal{Q}\left(A^{t} v\right)}{\mathcal{Q}(v)} \geq e^{2 a t} \quad \text { for } v \in \operatorname{int} C, \quad \frac{\mathcal{Q}\left(A^{t} v\right)}{\mathcal{Q}(v)} \leq e^{-2 a t} \quad \text { for } A^{t} v \in \operatorname{int} C^{\prime} .
$$

To compare the value of $\mathcal{Q}(v)$ with $\|v\|^{2}$ let us introduce the bundles of narrow cones $C_{1}=A^{1} C$ and $C_{1}^{\prime}=A^{-1} C^{\prime}$. By compactness, for some positive constants $c_{3}, c_{4}$ we get

$$
\mathcal{Q}(v) \geq c_{3}\|v\|^{2} \quad \text { for } v \in C_{1}, \quad-\mathcal{Q}(v) \geq c_{4}\|v\|^{2} \quad \text { for } v \in C_{1}^{\prime} .
$$


We introduce the intersection $C_{\infty}=\bigcap_{t \geq 0} A^{t} C$. In particular

$$
C_{\infty}(x)=\bigcap_{t \geq 0} A_{\phi^{-t} x}^{t} C\left(\phi^{-t} x\right) .
$$

Our goal is to show that $C_{\infty}(x)$ is the unstable subspace. Monotonicity yields the invariance property $A^{t} C_{\infty}=C_{\infty}$. Moreover since $\mathcal{Q}$ has constant signature $(l, m), C_{\infty}(x)$ must contain at least a subspace of dimension $l$. Indeed the $l$-dimensional subspaces contained in $A_{\phi^{-t} x}^{t} C\left(\phi^{-t} x\right)$ form compact subsets of the $l$-dimensional grassmannian, decreasing as $t$ increases. Hence their intersection is nonempty.

It follows from the inequalities above and the inclusion $C_{\infty} \subset C_{1}$ that for any $v \in C_{\infty}, t>0$, we have

$$
c_{2}\|v\|^{2} \geq \mathcal{Q}(v)=\mathcal{Q}\left(A^{t} A^{-t} v\right) \geq e^{2 a t} \mathcal{Q}\left(A^{-t} v\right) \geq c_{3} e^{2 a t}\left\|A^{-t} v\right\|^{2},
$$

i.e., $\left\|A^{-t} v\right\| \leq b e^{-a t}\|v\|$ for all $v \in C_{\infty}$ and some positive constant $b$. This decay property also holds in the linear span of $C_{\infty}$, perhaps with a different $b$.

Similarly starting with the negative cones $C^{\prime}$ we can define $C_{\infty}^{\prime}$ which over every point contains at least an $m$-dimensional subspace and $\left\|A^{t} v\right\| \leq$ $b e^{-a t}\|v\|$ for any $v \in \operatorname{span} C_{\infty}^{\prime}, t>0$.

It remains to show that $C_{\infty}(x)$ is an $l$-dimensional subspace and $C_{\infty}^{\prime}(x)$ is an $m$-dimensional subspace. This follows from the fact that the linear spans of $C_{\infty}(x)$ and $C_{\infty}^{\prime}(x)$ must be transversal. Indeed for any nonzero vector in the intersection we would have contradictory decay properties.

6. Proof of Theorem 4.1. Let us introduce the linearized equations for the general flow (3.1). Let $q(t ; u),|u|<\varepsilon$, be a family of trajectories for the system. We introduce the Jacobi field

$$
\xi=\frac{d q}{d u} \quad \text { and } \quad \eta=\frac{D v}{d u}=\frac{D}{d u} \frac{d q}{d t} .
$$

We get the following equations for $(\xi, \eta)$ (the linearization of the flow $(3.1))$ :

$$
\begin{aligned}
\frac{D \xi}{d t}= & \eta \\
\frac{D \eta}{d t}+R(\xi, v) v= & -\nabla_{\xi} \nabla W+\left(\nabla_{\xi} B\right) v+B \eta+\nabla_{\xi} E-\frac{\langle E, v\rangle}{v^{2}} \eta \\
& -\frac{1}{v^{2}}\left(\left\langle\nabla_{\xi} E, v\right\rangle+\langle E, \eta\rangle-\frac{2\langle E, v\rangle\langle v, \eta\rangle}{v^{2}}\right) v
\end{aligned}
$$

We introduce a quadratic form $\mathcal{H}$ on the tangent spaces of $T M$ by

$$
\mathcal{H}(\xi, \eta)=\frac{1}{2}\left(\xi^{2}-\frac{\langle\xi, v\rangle^{2}}{v^{2}}\right) .
$$


The form $\mathcal{H}$ can be naturally projected onto the quotient bundle (quotient by the span of the vector field (3.1)). We define $\mathcal{Q}$ as the Lie derivative of $\mathcal{H}$ in the direction of the vector field (3.1).

We get

$$
\begin{aligned}
\mathcal{Q}(\xi, \eta)= & \langle\xi, \eta\rangle-\frac{\langle\xi, v\rangle}{v^{2}}(\langle\eta, v\rangle-\langle\xi, \nabla W\rangle+\langle\xi, B v\rangle+\langle\xi, E\rangle) \\
& +\frac{\langle\xi, v\rangle^{2}}{v^{2}}\left(\langle E, v\rangle-\frac{\langle\nabla W, v\rangle}{v^{2}}\right) .
\end{aligned}
$$

To apply Theorem 5.2 we need to calculate the Lie derivative of $\mathcal{Q}$. This cumbersome task is simplified by restricting the resulting quadratic form to the subspace defined by

$$
\langle\eta, v\rangle=-\langle\xi, \nabla W\rangle, \quad\langle\xi, v\rangle=0 .
$$

The first equation corresponds to the restriction of the energy function $H=\frac{1}{2} v^{2}+W$, the second equation gives a representation of the quotient space. (Note that we can use the first equation before differentiating the form but the second equation can be used only after the differentiation.) Thanks to this restriction a considerable number of terms in $\frac{d}{d t} \mathcal{Q}$ vanishes. In particular we do not have to differentiate the terms in the first bracket and the terms in the second bracket give no contribution whatsoever to the restricted derivative. We obtain

$$
\begin{aligned}
\frac{d}{d t} \mathcal{Q}= & \eta^{2}-\langle R(\xi, v) v, \xi\rangle-\left\langle\xi, \nabla_{\xi} \nabla W\right\rangle+\left\langle\xi,\left(\nabla_{\xi} B\right) v\right\rangle+\langle\xi, B \eta\rangle \\
& +\left\langle\xi, \nabla_{\xi} E\right\rangle-\frac{\langle E, v\rangle}{v^{2}}\langle\xi, \eta\rangle \\
& -\frac{1}{v^{2}}\langle\xi,-2 \nabla W+B v+E\rangle^{2} \\
= & \left(\eta-\frac{\langle\eta, v\rangle}{v^{2}} v-\frac{\langle E, v\rangle}{2 v^{2}} \xi-\frac{1}{2} B \xi+\frac{\langle B \xi, v\rangle}{2 v^{2}} v\right)^{2} \\
& -\langle R(\xi, v) v, \xi\rangle-\left\langle\xi, \nabla_{\xi} \nabla W\right\rangle+\left\langle\xi,\left(\nabla_{\xi} B\right) v\right\rangle+\left\langle\xi, \nabla_{\xi} E\right\rangle \\
& -\frac{\langle E, v\rangle^{2}}{4 v^{4}} \xi^{2}-\frac{1}{4}(B \xi)^{2}+\frac{1}{4 v^{2}}\langle\xi,-2 \nabla W+B v\rangle^{2} \\
& -\frac{1}{v^{2}}\langle\xi,-2 \nabla W+B v+E\rangle^{2} .
\end{aligned}
$$

To end the proof of Theorem 4.1 we only need to check that the projected form $\mathcal{Q}$ is nondegenerate. We can represent the projected form by the restriction to the subspace (6.2). We obtain the form $\langle\xi, \eta\rangle$. It is straightforward to check that it is nondegenerate on the subspace (6.2). 


\section{References}

[A-S] D. V. Anosov and Ya. G. Sinai, Certain smooth ergodic systems, Russian Math. Surveys 22 (1967), 103-167.

[B-C-P] F. Bonetto, E. G. D. Cohen and C. Pugh, On the validity of the conjugate pairing rule for Lyapunov exponents, J. Statist. Phys. 92 (1998), 587-627.

[B-G-M] F. Bonetto, G. Gentile and V. Mastropietro, Electric fields on a surface of constant negative curvature, Ergodic Theory Dynam. Systems, to appear.

[D-M] C. P. Dettmann and G. P. Morriss, Proof of Lyapunov exponent pairing for systems at constant kinetic energy, Phys. Rev. E 53 (1996), R5541-5544.

[E-C-M] D. J. Evans, E. G. D. Cohen and G. P. Morriss, Viscosity of a simple fluid from its maximal Lyapunov exponents, Phys. Rev. A 42 (1990), 5990-5997.

[Go] N. Gouda, Magnetic flows of Anosov type, Tôhoku Math. J. 49 (1997), 165183.

[Gr] S. Grognet, Flots magnétiques en courbure négative, Ergodic Theory Dynam. Systems 19 (1999), 413-436.

[H] W. G. Hoover, Molecular Dynamics, Lecture Notes in Physics 258, Springer, 1986.

[K-B] A. Katok (in collaboration with K. Burns), Infinitesimal Lyapunov functions, invariant cone families and stochastic properties of smooth dynamical systems, Ergodic Theory Dynam. Systems 14 (1994), 757-786.

[K-H] A. Katok and B. Hasselblatt, Introduction to the Modern Theory of Dynamical Systems, Cambridge Univ. Press, 1995.

[L-Y] F. Ledrappier and L.-S. Young, The metric entropy of diffeomorphisms: I. Characterization of measures satisfying Pesin's formula, II. Relations between entropy, exponents and dimension, Ann. of Math. 122 (1985), 509-539, 540574.

[L] J. Lewowicz, Lyapunov functions and topological stability, J. Differential Equations 38 (1980), 192-209.

[P-P] G. P. Paternain and M. Paternain, Anosov geodesic flows and twisted symplectic structures, in: Dynamical Systems (Montevideo, 1995), Pitman Res. Notes Math. 362, Longman, 1996, 132-145.

[R] D. Ruelle, Positivity of entropy production in nonequilibrium statistical mechanics, J. Statist. Phys. 85 (1996), 1-23.

[V] I. Vaisman, Locally conformal symplectic manifolds, Internat. J. Math. Math. Sci. 8 (1985), 521-536.

[W] M. P. Wojtkowski, Systems of classical interacting particles with nonvanishing Lyapunov exponents, in: Lecture Notes in Math. 1486, Springer, 1991, 243-262.

[W-L M. P. Wojtkowski and C. Liverani, Conformally symplectic dynamics and symmetry of the Lyapunov spectrum, Comm. Math. Phys. 194 (1998), 47-60.

Department of Mathematics

University of Arizona

Tucson, AZ 85721, U.S.A.

E-mail: maciejw@math.arizona.edu

Received 28 July 1999;

in revised form 18 October 1999 